\title{
Shakespeare entre Nô et Kabuki : ou l'art dramatique élisabéthain cadré par Kurosawa Akira
}

Olivier Amour-Mayeur

\section{(2) OpenEdition}

12 Journals

Édition électronique

URL : http://journals.openedition.org/shakespeare/1513

DOI : 10.4000/shakespeare.1513

ISSN : 2271-6424

\section{Éditeur}

Société Française Shakespeare

\section{Édition imprimée}

Date de publication : 13 décembre 2009

Pagination : 137-158

ISBN : 2-9521475-6-6

\section{Référence électronique}

Olivier Amour-Mayeur, « Shakespeare entre Nô et Kabuki : ou l'art dramatique élisabéthain cadré par

Kurosawa Akira », Actes des congrès de la Société française Shakespeare [En ligne], 27| 2009, mis en ligne le 13 décembre 2009, consulté le 21 avril 2019. URL : http://journals.openedition.org/ shakespeare/1513 ; DOI : 10.4000/shakespeare.1513 


\title{
Shakespeare et l'Orient
}

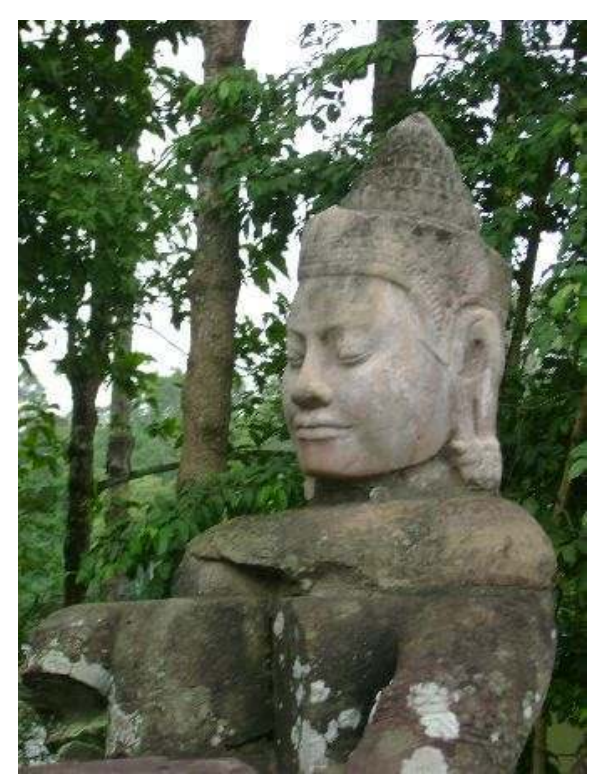

\author{
actes du Congrès \\ organisé par la \\ les 12, 13 et 14 mars 2009 \\ textes réunis par \\ Pierre KAPITANIAK \\ sous la direction de \\ Jean-Michel DÉPRATS
}

SOCIÉTÉ FRANÇAISE SHAKESPEARE 
COUVERTURE :

photo J.-M. Déprats 2008

conception graphique et logo

Pierre Kapitaniak

\section{(C) 2009 Société Française Shakespeare \\ Institut du Monde Anglophone \\ Université de Paris III - Sorbonne Nouvelle \\ 5 rue de l'École de Médecine 75006 Paris \\ www.societefrancaiseshakespeare.org}




\title{
SHAKESPEARE ENTRE Nô ET KABUKI : OU L'ART DRAMATIQUE ÉLISABÉTHAIN CADré Par KurosaWa AKIra
}

\author{
Olivier AMMOUR-MAYEUR
}

Quels liens peut-on tisser entre l'œuvre de Shakespeare et l'art cinématographique japonais ? C'est à cette question que tente de répondre cet article, d'abord en soulignant les analogies étonnantes qui se font jour entre les scènes élisabéthaine et japonaise, ensuite en analysant certains éléments dramaturgiques tirés de Macbeth, adapté dans Kumonosujo (Le Château de l'araignée), par « l'empereur » du cinéma japonais. Cette confrontation a pour ambition de montrer comment certains déplacements narratifs opérés par le réalisateur, d'une part, et comment l'usage des arts théâtraux japonais, d'autre part, renforcent l'effet tragique de la pièce shakespearienne, sans en trahir l'essence.

What connections may one establish between Shakespeare's work and Japanese cinematographic art? The present paper will try to answer this question, first by underlining the astonishing analogies that appear between Elizabethan and Japanese stages and then by analysing some dramatic elements taken from Macbeth, adapted in Kumonosujo (Throne of Blood), by the "emperor" of Japanese cinema. This confrontation aims at showing how some narrative displacements worked by the director on the one hand, and the use of Japanese dramatic arts on the other strengthen the tragic effect of Shakespeare's plays, without betraying their essence.

S'il faut de l'irreprésentable pour composer la représentation, n'est-ce pas parce que le poids de l'ombre constitue un espace inassignable, un entre-espace flottant comme les superpositions cinématographiques ? Comment saisir une ombre? Comment étreindre un fantôme sinon dans l'aporie renouvelée d'un être en retrait et effacement, d'une loi qui se redouble?

Christine Buci-Glucksmann, Tragique de l'ombre, p. 30.

l'instar d'un Magritte inscrivant sur ses toiles «Ceci n'est pas
une pipe », certains critiques puristes ont clamé devant les
adaptations de Kurosawa « ceci n'est pas du Shakespeare »,
alors même que l'image du réalisateur souligne combien le dramaturge
n'est absolument pas trahi par ce travail cinématographique. Pour
mettre au jour les liens saisissants qui unissent dramaturgie
élisabéthaine et arts de la scène japonais, cet article s'en tiendra à
l'analyse du Château de l'araignée, car une lecture de Ran, dans cette
perspective, se révèlerait redondante, Kurosawa ayant toujours
procédé selon les mêmes principes esthétiques pour ses adaptations 
shakespeariennes. En fin d'article, cependant, un exemple prélevé sur Ran tentera de mettre cette systématicité en évidence.

Au préalable, il s'agit de montrer que l'art théâtral Nô se rapproche par bien des traits de celui de la scène anglaise (dans ses dispositifs scéniques comme dans la composition des troupes d'acteurs), ensuite il s'agit de souligner le fait que ces relations trouvent en partie leurs racines dans la pensée antique, puisque Shakespeare s'est inspiré des enseignements de Sénèque, philosophe stoïcien, dont la pensée s'apparente, à bien des égards, à celle du bouddhisme Zen, notamment dans ses visées éthiques ${ }^{1}$; nous y reviendrons plus loin.

Enfin, le dernier point tente de mettre en évidence le fait que si Kurosawa, dans ses adaptations, ne peut pas toujours respecter la lettre shakespearienne, il garde cependant à cœur d'en respecter l'essence, et surtout que son détour par le Nô et le Kabuki, dans son travail de mise en scène, ne se fait qu'en trahissant du même geste ces deux autres formes d'art dramatique. En d'autres termes, si trahison il y a, celle-ci porte en fait sur certains aspects externes des genres théâtraux sollicités par le réalisateur, et toujours dans le plus grand respect observé envers l'esprit de la tragédie shakespearienne et celui des arts théâtraux japonais.

\section{Espaces scéniques : Angleterre/Japon}

Si l'on s'attache à une comparaison des genres dramaturgiques, une fois dépassés les différences de surface, il appert que certains éléments ne changent guère, et ce quel que soit le pays. En Angleterre comme sur le continent européen, mais non moins au Japon, le théâtre nait d'une contradiction formant double bind. D'un côté ce sont les rites religieux sécularisés, produisant les miracles et mystères en Occident et les libations autochtones au Japon, qui ouvrent la voie de son devenir, de l'autre les acteurs sont tous considérés comme des parias,

\footnotetext{
${ }^{1}$ Pour mettre les deux pensées en confrontation, voir sur le bouddhisme : Damien Keown, Buddhist Ethics - A Very Short Introduction, Oxford, O.U.P., « Very Short Introductions », 2005 et, pour une étude plus poussée : Peter Harvey, An Introduction to Buddhist Ethics : Fondations, Values and Issues, Cambridge, C.U.P., 200o. Sur le stoïcisme, voir notamment: The Cambridge Companion to The Stoics (Brad Inwood Ed.), Cambridge, C.U.P., 2003, plus particulièrement chap. 7 "Stoic determinism » (par Dorothea Frede) p. 179-205 et chap. 9 « Stoics Ethics » (par Malcholm Schofield), p. 233-256.
} 
apparentés aux vagabonds, aux malfaiteurs et aux prostituées ${ }^{2}$. La sécularisation du théâtre ayant oublié ses origines religieuses, les acteurs japonais, comme européens, afin de se prémunir des attaques religieuses, et parfois populaires, sont ainsi amenés à rechercher la protection des nobles, seuls à être suffisamment puissants et craints pour leur apporter soutien financier et protection.

La scène du Nô, au même titre que la scène anglaise, a gardé les traces de ses origines, puisque de nos jours encore, elle conserve l'architecture de l'époque où les pièces étaient jouées en plein air, notamment dans les sanctuaires shinto ou les temples bouddhistes. Bien entendu un élément important joue dans la conservation de cet ensemble architectural, c'est le caractère tout utilitaire des piliers présents sur scène, qui servent de points de repère à l'acteur qui porte un masque dont les ouvertures pour les yeux sont très étroites.

Cependant, les ressemblances entre scènes anglaises et japonaises ne s'arrêtent pas là. En effet, en Angleterre, contrairement aux autres scènes européennes, aucun rideau ne vient séparer l'audience du proscenium. De même, aucun décor proprement dit n'est utilisé lors des représentations. Henri Fluchère ajoute, dans sa description de la scène anglaise: "On utilisait cependant des accessoires (properties) à la fonction symbolique, pour créer une illusion de réalisme, et les lieux scéniques pouvaient être figurés par des pancartes, ou indiqués dans le texte ${ }^{3}$. " Il en va de même sur la scène de Nô (le système, en revanche, s'est développé différemment en ce qui concerne le Kabuki). La scène est nue, si ce n'est le sapin tortueux peint en fond de scène, présent pour symboliser la nature (les

\footnotetext{
${ }^{2}$ Voir le chapitre «Le théâtre anglais » de Henri Fluchère, dans Histoire des spectacles, dir. Guy Dumur, Paris, Gallimard, coll. Encyclopédie de la Pléiade, 1965, p. 690-738. Il faut préciser ici qu'en Europe comme au Japon ce sont des acteurs amateurs, en général les novices attachés à l'église ou au temple, qui interprètent les Mystères ou les Libations. Les acteurs, au sens professionnel du terme, sont dans tous les cas mal perçus car appartenant, tout du moins au départ, à la catégorie des itinérants. Sans domicile fixe, ils sont ainsi considérés par les autorités comme fauteurs de trouble possibles. Sur ce point, du côté anglais, voir R. A. Foakes, "Playhouses and players ", in The Cambridge Companion to English Renaissance Drama (éds. A. R. Braunmuller \& Michael Hattaway), $2^{\mathrm{e}}$ éd., Cambridge, C.U.P., 2003, p. 2.

3 Henri Fluchère, op. cit., p. 709. Bien qu'ancienne, l'analyse proposée par Fluchère n'est remise en cause dans aucun des autres ouvrages consultés pour cet article. En plus de l'étude de Foakes déjà citée, on peut voir Andrew Gurr, The Shakespearean Stage (15741642), 4 e éd., Cambridge, C.U.P., 2009.
} 
spécialistes ${ }^{4}$ pensent qu'il s'agit d'un vestige référentiel de l'époque où le Nô se jouait à l'extérieur), mais surtout afin de métaphoriser l'axe du monde, reliant le monde des vivants et celui de l'au-delà. Les lieux ne sont pas représentés, ils sont indiqués soit par le titre du Nô lui-même, soit par les dialogues. Les accessoires, là aussi, sont sommaires : les manches du costume de l'acteur, un éventail, qui devient, en fonction des besoins, ombrelle, poignard, plateau, sabre... Cet éventail sert, de même, à l'expression des sentiments éprouvés par les personnages. Enfin, élément important de la dramaturgie, le masque porté par le Shite (personnage principal) qui ne sert pas uniquement comme effet esthétique, mais s'avère nécessaire en tant que lieu même où s'incarne le caractère (et non personnage) ${ }^{5}$ que joue l'acteur (le plus souvent un fantôme, un démon ou un dieu).

En ce qui concerne le jeu des acteurs, il existe aussi plusieurs points de similitudes entre les deux scènes. Henri Fluchère souligne notamment que « les costumes étaient riches et soignés, exécutés dans les plus beaux tissus, et faisaient la gloire de l'acteur' ${ }^{6}$. » Du côté de la scène japonaise, jusqu'aujourd'hui les costumes sont exclusivement faits de soie et de tissus précieux. Le costume a une importance certaine, étant donné que l'acteur évoluant sur scène ne bouge que très peu, de façon hiératique et selon une économie de moyens qui tend, dès lors, à attirer l'attention du public sur les moindres changements subis par le costume qu'il porte, en fonction des reflets de lumière et des gestes de l'acteur. À travers cette esthétique du peu, ce sont toutes les déclinaisons de l'infinitésimal qui prennent de l'importance. Les mouvements les plus délicats servant à exprimer la peur, la tristesse, la colère, doivent donc être perçus autrement que par la brusquerie des

\footnotetext{
4 Voir par exemple Donald Keene, Nô \& Bunraku, New York, Columbia University Press, $2^{\mathrm{e}}$ éd., 1990 ; Eric C. Rath, The Ethos of Noh, Actors and Their Art, Cambridge, Harvard University Press, "Harvard University Asia Center », 2004 et René Sieffert, Théâtre classique, Paris, P.O.F., « Arts du Japon », $2^{\mathrm{e}}$ éd., 1997.

5 Il est nécessaire de distinguer le « caractère » du théâtre Nô du «personnage » du théâtre occidental, car il n'est jamais question, dans le premier cas, d'incarner un être pétri de «psychologie », encore moins de lui donner un « visage » et des expressions lui imprimant un tempérament qui varierait selon les mises en scène. Le «caractère » Nô renvoie davantage au sens étymologique du terme français : "propre à un style », style étant ici à entendre au sens de "style théâtral ». La performance d'un même caractère étant immuable d'une représentation à l'autre, voire d'une pièce à l'autre: par exemple, le fantôme répond à une codification du jeu scénique rigoureuse, quelle que soit la pièce. Voir le Dictionnaire historique de la langue française (Dir. Alain Rey), 1991, rééd. 1998, vol. I, p. 622.

${ }^{6}$ Henri Fluchère, op. cit. p. 709.
} 
gestes, généralement absente, ou par l'expression du visage, puisque ce dernier est caché derrière un masque ; à moins qu'il ne reste impavide, selon les recommandations aux acteurs, s'il est découvert.

Pour ce qui est de la musique, un autre parallèle peut être tracé. $\mathrm{Si}$, en Angleterre, il est considéré que «la musique était [...] intimement liée à l'action, en soulignant le lyrisme et la passion, ou l'agrémentait de ses arabesques et de ses chansons. Son rôle était moins d'accompagnement que de suggestion active, elle ajoutait ses sortilèges à ceux du verbe 7 », on peut alors rapprocher cette fonction de celle que René Sieffert attribue à la musique du Nô : «Le rôle de [1']orchestre est essentiel. Non qu'il s'agisse d'une véritable musique d'accompagnement : ce serait plutôt un bruitage rythmé, destiné à créer l'atmosphère propice à l'évocation de tel ou tel type de personnage $^{8}$. » Si les deux fonds sonores restent sensiblement distincts dans leur performance, comme dans leur acoustique, il reste cependant important de souligner que dans chaque cas la musique n'accompagne pas le jeu, elle signifie autrement, autre chose, et, dans le Nô pareillement, ajoute « ses sortilèges à ceux du verbe ».

Pour ce qui concerne le rythme dramaturgique de la pièce, un nouveau parallèle intéressant mérite toute l'attention. On sait que sur les scènes européennes, et notamment dans la tragédie, le tempo suit une scansion ternaire depuis son commencement jusqu'à la résolution de «la crise » finale : protase, acmé, apodose. La protase étant le moment du « coup d'envoi » du drame, l'acmé son « point d'intensité » et l'apodose sa « résolution». Le théâtre japonais repose sur cette même scansion ternaire dans son travail de tension dramatique. Les trois termes clés de la dramaturgie Nô sont: Jo, Ha, Kyû. Zeami reprend en fait une règle ancienne de l'esthétique japonaise car, ainsi que le formule Armen Godel, « les trois termes de cette triade illustrent le phénomène de la progression qui sous-tend toute chose » 9 . On peut traduire ces termes ainsi :

Jo : début / attaque / lent ;

Ha : développement (déroulement) / cassure (rupture) / modéré

\footnotetext{
7 Henri Fluchère, Ibid., je souligne.

${ }^{8}$ René Sieffert, in Zeami, La Tradition secrète du Nô (trad. René Sieffert), Paris, Gallimard/Unesco, coll. Connaissance de l'Orient, 1960, p. 17, je souligne.

${ }^{9}$ Armen Godel, Le Maître de Nô, Paris, Albin Michel, coll. Question de, 1989, rééd. coll. Espaces Libres, 2004, p. 122.
} 
(moyen) ;

Kyû : fin (dénouement) /final / rapide (précipité) ${ }^{10}$.

S'il faut nuancer un peu le rapport de tempo entre les deux cultures théâtrales, étant donné que le rythme ternaire japonais se développe en fait en cinq étapes, tout en étant ternaire ${ }^{11}$, il reste cependant que l'esthétique théâtrale semble provoquer d'elle-même ces éléments dramaturgiques dont on considère, depuis, qu'ils fondent le Sublime des arts de la scène japonaise. Bien entendu, il n'est nullement dans mon intention de nier les spécificités de chaque art scénique, cependant, il semble important d'en passer par une analyse quelque peu structuraliste de ces formes, afin de pouvoir en apprécier les variantes.

Enfin, avant de passer à l'analyse de la mise en scène proprement dite, il reste à rappeler que sur les deux scènes, et bien que le règne de Jacques $\mathrm{I}^{\mathrm{er}}$ fasse évoluer les choses en la matière, seuls les hommes sont autorisés à jouer. Au Japon, les rôles de femmes deviendront, avec le temps, la spécialité des onnagata, acteurs qui, parfois, et ce jusqu'à l'ère Meiji, vivaient en femme même sortis de scène. Cet élément dramaturgique, qui peut a priori sembler anodin aujourd'hui, revêt en fait une importance capitale, notamment en ce qui concerne les rôles de fantômes (ou sorcières). Car si nous savons que Shakespeare jouait avec la question des genres dans son théâtre, grâce à cette tradition des acteurs jouant les rôles féminins, nous verrons plus loin que Kurosawa joue sur cet aspect dans son travail d'adaptation, tout en détournant quelque peu l'usage de la tradition japonaise.

\section{Esthétiques de la mise en scène : Sénèque / Shakespeare / Kurosawa}

La comparaison peut s'étendre au travail de mise en scène, telle qu'elle était pratiquée à l'époque de Shakespeare, et celle de ses contemporains japonais. Bien entendu, il ne peut s'agir ici de faire un inventaire complet des techniques de jeu des acteurs. Cependant,

\footnotetext{
10 Ibid.

${ }^{11}$ Le rythme reste ternaire car les cinq pièces représentées lors de la journée de Nô se déploient toujours selon le même principe : une pièce Jo en introduction, deux pièces Ha en développement et une pièce Kyû en conclusion.
} 
quelques pistes peuvent déjà indiquer les croisements qui s'opèrent entre « l'art de la fleur ${ }^{12}$ » japonais et les principes de jeu de l'époque shakespearienne. Cette comparaison est facilitée par le fait que les pratiques du théâtre Nô n'ont quasiment pas évolué depuis leur âge d'or, au XVII siècle. Ainsi, si l'on reprend les propos d'Henri Fluchère en ce qui concerne les pratiques du théâtre élisabéthain, Zeami et ses descendants n'auraient sans doute rien à y redire :

L'acteur devait [...] travailler sa voix et son articulation, et aussi ce que tous les traités de rhétorique appellent l'action, c'est-à-dire ses gestes, son maintien, sa mimique. Tous ces mouvements étaient (nous le savons maintenant) régis par des conventions précises dont les chirologies et les chironomies de l'époque nous donnent quelque idée. Chaque émotion violente avait sa symbolique de gesticulation à laquelle il fallait se conformer. On ne s'improvisait pas acteur ${ }^{13}$.

De même, on ne s'improvisait pas acteur de Nô. D'où le fait, entre autre, que l'apprentissage commence au plus tôt, puisque Zeami donne des indications de jeu pour des acteurs âgés de sept ans. En ce qui concerne la gestuelle elle-même, celle-ci est décrite de façon précise, rôle par rôle, dès le livre II du traité du maître. Le dramaturge explique notamment, en ce qui concerne les rôles «à visage découvert », qu'étant « un spectacle intolérable », le fait de modifier «son expression habituelle» est sans doute l'élément le plus intolérable. Zeami en conclut que «c'est par son comportement, par son allure, qu'il faut imiter son personnage ${ }^{14}$, » le visage devant, lui, rester impassible. De façon plus explicite encore, pour les rapprochements possibles avec le théâtre élisabéthain, la description du jeu du démon nous éclaire davantage sur les attentes du maitre de Nô :

Si l'on interprète le hataraki les yeux fixés sur son partenaire, en exécutant minutieusement les mouvements des mains et des pieds, et en prenant appui sur le rythme de la percussion, l'intérêt y trouve un support. Si l'on mimait fidèlement un authentique démon des Enfers, l'intérêt ferait totalement défaut, car il serait simplement horrible ${ }^{15}$.

\footnotetext{
12 «L'art de la fleur » est la traduction littérale de Fûshi Kaden, nom du traité secret de Zeami qui transmettait l'art théâtral Nô de génération en génération.

13 Henri Fluchère, op. cit., p. 713.

14 Zeami, La Tradition secrète du Nô, op. cit., p. 72, souligné dans le texte.

15 Ibid., p. 74
} 
C'est donc uniquement à travers la gestuelle du rôle que le caractère prend tout son sens et sa puissance. Nulle trace, dans cet art, de « réalisme » de jeu, au sens où on l'entend aujourd'hui. Dès lors, dans les deux écoles, la gestuelle répond à une codification précise qui ne laisse pas de place à l'improvisation ${ }^{16}$. Ainsi, tendre « un miroir à la nature », selon l'appel lancé par Hamlet lorsqu'il parle aux acteurs, ne revient pas à « imiter » celle-ci. Au contraire, le dessein du dramaturge, parlant à travers son personnage, est plutôt d'en révéler ainsi l'essence et non son simple reflet, à l'instar de ce que préconise l'art du Nô selon d'autres principes. Hamlet exige en effet de ses acteurs certaines règles de jeu qu'il semble intéressant de rappeler :

HAMLET. Speak the speech, I pray you, as I pronounc'd it to you, trippingly on the tongue ; but if you mouth it as many of our players do, I had as lief the town-crier spoke my lines. Nor do not saw the air too much with your hand, thus, but use all gently : for in the very torrent, tempest, and as I may say, whirlwind of your passion, you must acquire and beget a temperance that may give it smoothness [...]

Be not too tame, neither ; but let your own discretion be your tutor. Suit the action to the word, the word to the action, with this special observance, that you o'erstep not the modesty of nature. For anything so o'erdone is from the purpose of playing, whose end, both at the first and now, was and is to hold as 'twere the mirror up to nature, to show virtue her own feature, scorn her own image, and the very age and body of the time his form and pressure. (Hamlet, III.ii.1-24)

Le travail réclamé sur le geste et l'alliance de ce dernier avec le mot permettent un nouveau rapprochement avec la pratique du théâtre Nô. Zeami, dans une autre partie de son enseignement, rappelle en effet comment la voix et le geste doivent se coordonner, afin de soulever l'« intérêt » du public :

Dans toute mimique, la manière est l'interprétation d'un texte par le geste. Or, il arrive que l'interprétation [par le geste] coïncide avec le texte, mieux, que l'allure devance la parole. Audition et vision se trouvent interverties. Si l'on fait précéder ce que saisit l'oreille du public, et que l'on interprète [donc] de façon à retarder légèrement l'allure, à l'instant précis où, à l'impression auditive se substitue la vision, naît le sentiment que l'interprétation est parfaite ${ }^{17}$.

${ }^{16}$ Pour plus de détails, voir Andrew Gurr, The Shakespearean Stage.

${ }^{17}$ Zeami, op. cit., p. 116. 
Faire voir juste avant de dire permet ainsi d'atteindre le summum de l'art théâtral. La parfaite coordination entre les deux éléments du jeu rappelle, ainsi, que rien ne relève de l'aléatoire ou de l'improvisation. Dès lors, c'est bien toute la combinatoire d'une pratique, d'un entraînement, entre geste, parole et musique, qui fait que l'art du théâtre retient l'attention du spectateur.

Avant de passer à l'analyse des films de Kurosawa, il reste encore à analyser pourquoi les arts théâtraux classiques de la scène élisabéthaine et ceux du Japon se retrouvent sur ces points plus proches que ceux des autres scènes européennes classiques. Dans cette perspective, il est probable que l'influence de Sénèque sur le travail de Shakespeare, et plus largement la redécouverte du stoïcisme à son époque, en soit l'une des raisons ${ }^{18}$. Par conséquent, la mise en parallèle de la philosophie stoïque et de la philosophie bouddhique, dont les principes coïncident en plus d'un point, permet d'expliquer, du moins en partie, pourquoi le plus grand dramaturge du Bunraku et du Kabuki (Chikamatsu Monzaemon, 1653-1724) est depuis longtemps surnommé le «Shakespeare japonais », d'autre part, pourquoi les tragédies de Shakespeare sont autant adaptées, et mises en scène sur le sol japonais. Le dramaturge anglais, en effet, puise une grande partie de son inspiration aux textes latins de Sénèque qui est apparenté au stoïcisme tardif. Il s'agit non seulement d'une philosophie préchrétienne, mais surtout certains traits de cette philosophie sont très proches de la philosophie bouddhique, notamment dans sa version Zen, la plus répandue au Japon. Les deux mouvements sont polythéistes, croient aux forces du Destin (Fatum) - il y en a plusieurs -, ont une vision cyclique du temps, et non linéaire (issue de la notion du Destin et qui implique celle d'un «éternel retour»), et surtout une certaine conception de la réincarnation revêt pour les deux mouvements une grande importance, ce qui implique que l'existence est régie par un système où toute action accomplie entraîne " rétribution », en bien ou en mal. C'est cette notion, notamment, qui apporte au théâtre japonais sa cohorte de démons et de fantômes errants. N'ayant pas trouvé le

18 Pour des analyses plus précises, et qui vont dans le sens de notre interprétation, voir : Robert S. Miola, Shakespeare and Classical Tragedy. The Influence of Seneca, Oxford, Clarendon Press, 1992 et Shakespeare and the Classics (éd. Charles Martindale \& A. B. Taylor), Cambridge, C.U.P., 2004, notamment les chapitres de Yves Peyré, "Senecan resonances in Macbeth », p. 141-155 et d'Erica Sheen, "These are the only men' : Seneca and Monopoly in Hamlet 2.2 ", p. 156-167. 
repos en raison de leur vie passée sur terre (ils sont souvent en quête d'une vengeance ou d'un pardon), ils errent en attendant l'accomplissement du geste qui leur permettra de passer dans l'au-delà. Or, les fantômes ont une importance capitale dans le théâtre shakespearien. Même si l'on peut douter que Shakespeare adhérait aux croyances du philosophe qui l'inspirait, l'usage qu'il fait des revenants et des êtres surnaturels dans ses pièces montre que ces derniers servaient son propos éthique, ainsi que son travail de dramaturge.

Pour mettre en évidence les nœuds exemplaires qu'il est possible de nouer entre Stoïcisme et Bouddhisme, un exemple permettra de mettre au jour les principes similaires qui prévalent dans l'une et l'autre école. Si nous suivons le cas de Macbeth (Washizu chez Kurosawa), cette figure parait exemplaire de deux traits fondamentaux qui sous-tendent les deux écoles antiques. D’un côté, la croyance dans un Destin (ou Fatum) qui oriente l'existence de tout être humain, de l'autre, et aussi paradoxal que cela puisse paraître avec la première assertion, la liberté d'action qui garde la prééminence dans le Destin de l'individu. Ce que Peter Harvey explique en ces termes :

While belief in the law of karma can sometimes degenerate into a form of fatalism, the Buddha emphasized that deterministic fate (niyati) and karma are very different. The idea of karma emphasizes the importance of human action and its effects : people make their own 'destiny' by their actions. Karma and fatalism differ on two scores. Firstly, humans have freedom of choice; their present actions are not the karmic results of previous actions, though karmic results may influence the type of action that a person tends to think of doing, because of the character he or she has developed. Secondly, not everything that happens to a person is seen as due to karma. Any unpleasant feelings or illnesses that one has can arise from a variety of causes $[\ldots]^{19}$.

Avant de poursuivre, il est important de souligner que pas plus que le Karma bouddhique, le Fatum latin n'a de lien direct avec la définition du terme «fatalisme » contemporain. C'est ce que rappelle Dorothea Frede :

When Chrysippus [troisième fondateur du stoïcisme] is concerned with the general picture, he emphasizes that nothing happens outside the allencompassing ordinance of the casual order of fate so that nothing can turn out other than in accordance with that ineluctable order [...]. When it comes to the explanation of individual events, the perspective changes, and it turns out that 'fate' is not a uniform concatenation of

19 Peter Harvey, op. cit., p. 22. 
necessitating antecedent causes, but rather a web that contains quite different elements with different functions and powers. ${ }^{20}$

Ainsi, si l'on rapporte ces deux considérations sur le Fatum au personnage de Macbeth, il devient clair que Shakespeare construit ce personnage selon une perspective toute stoïque, à laquelle s'agrège parfaitement la figure de Washizu son «descendant» japonais. En effet, si d'après les deux propositions on peut considérer, selon une perspective simpliste, que le Destin de Macbeth est noué dans sa rencontre avec les sorcières : ce serait elles qui le pousseraient à agir comme il le fait dans la suite de la pièce, il ne serait donc pas totalement responsable. Après lecture des deux analyses proposées par les deux spécialistes, il s'avère, tout au contraire, que Macbeth est responsable de ses actes et, donc, de ses crimes. Il aurait pu aussi bien choisir une autre voie sans céder au chant tentateur du pouvoir. Par ailleurs, l'analyse proposée par Dorothea Frede nous intéresse ici à double titre, puisqu'elle conclut sur l'idée d'une «toile d'éléments différents » qui fait signe vers le titre donné à son film par Kurosawa (Kumonosu Jô/The Cobweb Castle/Le château de l'araignée). Titre par lequel Kurosawa entendait donc rendre l'idée d'un faisceau de circonstances dans lequel finit par s'empêtrer Washizu/Macbeth avant que son comportement n'engendre sa chute.

Par suite, il est possible d'affirmer que deux des adages les plus connus du Fatum shakespearien ne seraient pas reniés par les bouddhistes Zen. D'abord celui proféré par le Jaques d'As You Like It et dont Shakespeare a fait la devise du Globe :

All the world's a stage,

And all the men and women merely players :

They have their exits and their entrances;

And one man in his time plays many parts..

(II.vii.147-150)

Ce « monde-scène », dont parle la formule de Shakespeare, est d'une certaine façon celui mis en image dans les ukiyo-e ( « images du monde flottant »), les célèbres estampes japonaises, qui cherchent à donner un aperçu de ce monde d'illusions où les hommes ne font que passer et où leur "divertissement » (au sens pascalien du terme) a pour seul objet de faire passer le temps, avant de faire «leur sortie » (et éventuellement de revenir pour une nouvelle existence).

${ }^{20}$ Dorothea Frede, art. cit., p. 199. 
Une autre formule est celle proférée par Richard III dans la pièce éponyme, lorsqu'il s'écrie : «Atteindre au sommet c'est rouler dans l'abîme ${ }^{21}$. » À travers le topos de la chute des princes et de la fortune qui tourne, cette sentence rejoint, à sa façon, la notion d'« éternel retour » selon les vues extrême-orientales, puisque celle-ci implique davantage le principe d'un cycle transformateur qu'un simpliste retour du même immuable. L'homme grimpant les échelons $\mathrm{du}$ pouvoir ne peut qu'arriver, à un certain moment, au faite de sa carrière. Cependant parvenir à ce point implique, dans un même mouvement, qu'il ne peut, à partir de là, que redescendre. Plus proche encore pour ce qui concerne les liens avec les adaptations de Kurosawa, dans King Lear, le fils bâtard et traître Edmond a ce propos au moment où son complot est déjoué et qu'il agonise : "The wheel is come full circle, I am here. » (v.iii.165). Toutes ces maximes, ne l'oublions pas, ont été formulées en des temps où le pouvoir, d'un côté comme de l'autre du globe, était des plus instables. Ainsi, les dramaturges tentaient, selon leurs moyens, de moraliser le pouvoir politique qui leur était contemporain. Étant donné les alliances, trahisons et retournements d'alliances, ces préceptes n'avaient ainsi aucun mal à dire vrai, puisque les seigneurs de guerre, anglo-saxons comme japonais, dont les pièces nous parlent, sont pris dans la toile tragique de leur existence. Et qui dit tragédie, dit déchéance, d'une façon où d'une autre.

\section{De la trahison comme fidélité : Kurosawa entre théâtre élisabéthain et scènes japonaises}

En ce qui concerne les adaptations de Kurosawa, il faut préalablement rappeler qu'à l'instar du cinéma européen, le cinéma japonais a d'abord été considéré comme un prolongement de l'art théâtral (contrairement à la tradition américaine, où l'histoire du théâtre était beaucoup moins ancrée) et qui a d'emblée assimilé le cinéma à l'art photographique. Il aura fallu un certain temps avant que les cinéastes japonais se dégagent de la chape de la «tradition » et parviennent à inventer leur propre esthétique. Or, durant son enfance et son adolescence, Kurosawa a été nourri principalement de films américains. Cette

${ }^{21}$ Formule citée par Christine Buci-Glucksmann, in Tragique de l'ombre, Paris, Galilée, « Débats ", 1990,, p. 87. 
formation par la salle de projection a eu un impact certain sur son parcours ultérieur de réalisateur. C'est sans doute pourquoi l'industrie cinématographique japonaise a toujours eu du mal à accepter le maître dans son cercle, en considérant qu'il était un cinéaste "pour occidentaux ». Ainsi, ce n'est sans doute pas un hasard non plus s'il a toujours eu à dessein d'adapter Shakespeare, dont il admirait l'œuvre, et dont trois pièces ont inspiré ses scénarios.

Si Hamlet a nourri de façon très libre l'univers de Kurosawa (beaucoup ne savent pas, aujourd'hui encore, que la pièce se trouve à l'origine de Les Salauds dorment en paix, 1960), il n'en reste pas moins vrai, cependant, et ce malgré les critiques formulées contre Kumonosu Jô (1957) ou Ran (1985), que ces derniers rendent un tribut spectaculaire à l'art dramatique shakespearien. Afin de le mettre en évidence, il est nécessaire de confronter le texte des pièces non seulement aux dialogues des films mais surtout à leurs images. Kurosawa, parmi les premiers, a toujours refusé de faire du théâtre filmé et a surtout toujours rejeté ce «style», toujours considéré comme passéiste et insupportable. C'est donc toute la problématique de l'adaptation - au sens de traduction - qui s'est imposée au réalisateur, lorsqu'il a cherché à mettre en scène Macbeth $^{22}$.

Certains critiques contemporains du cinéaste ont réprouvé le film, au motif que la langue de Shakespeare n'était pas respectée. Mais la langue japonaise est si radicalement différente des langues occidentales qu'une telle tentative est vouée à l'échec, à moins de tenter de transposer la poétique de la langue d'origine dans celle d'arrivée, autrement dit en faisant appel à la poétique de la langue théâtrale japonaise (qu'il s'agisse du Nô ou du Kabuki). Or, c'est précisément ce qu'a tenté le réalisateur, non à travers les dialogues - au risque sinon de refaire du théâtre filmé - mais plutôt à travers la transposition de la composition d'ensemble. Et c'est bien le pari qu'a tenté Kurosawa, en intégrant à sa mise en scène des éléments de Nô, mais aussi - à son corps défendant - de Kabuki. Or, si Kurosawa utilise certains éléments de la dramaturgie du Nô, il le fait de la même façon que pour le texte shakespearien, c'est-à-dire en trahissant sa «théâtralité », afin d'en

\footnotetext{
22 Pour d'autres analyses stimulantes, mais qui laissent de côté l'apport de la culture japonaise, sur cette adaptation, voir l'article synthétique de Serge Chauvin, in Shakespeare et le cinéma, éd. Jean-Marie Maguin \& Patricia Dorval, Société Française Shakespeare, Congrès 1998, p. 31-35.
} 
conserver l'essence et les éléments essentiels à la mise en scène d'une tragédie cinématographique.

L'un des éléments centraux sur lequel s'est longuement interrogé le réalisateur concerne les sorcières, «The Weird Sisters". Car quand bien même les fantômes et autres esprits vengeurs sont légion au Japon, aucune figure de sorcière telle que décrite par Shakespeare n'y est plausible. Le réalisateur en témoigne dans une interview donnée à son ami Donald Richie en 1960 :

The problem was: How to adapt the story to Japanese thinking. The story is understandable enough but the Japanese tend to think differently about such things as witches and ghosts. [Though ghosts tend to be vengeful, as in innumerable kaidan films, the idea of a gratuitously malevolent trio of witches is far from the Japanese imagination $]^{23}$.

Ainsi, la pièce s'ouvrant sur l'apparition des «Weird Sisters » (scène i) et la rencontre de Duncan avec un soldat blessé qui vient lui relater les événements de la « bataille gagnée-perdue ${ }^{24}$ » (scène ii), le sentiment d'étrangeté que cet enchaînement provoque est déplacé dans le film vers celui des arrivées successives de quatre soldats, aux messages contradictoires, au Château de l'araignée où le Seigneur (Fujimaki/Duncan) réside. Le déplacement (trois plus un dans la pièce $v s$ un plus trois dans le film) installe la tension dramatique d'une façon équivalente : le chiffre et la multiplicité des discours tenus rappelle qu'il faut se méfier des messages que l'on reçoit. D'ailleurs, dans le film, la succession rapide des messagers (orchestrée à travers un travail vif des raccords) permet d'insister sur le fait qu'un chef digne de ce nom doit prendre le temps de la réflexion avant d'agir - contrairement à ce que Washizu/Macbeth mettra en pratique plus tard.

Plus important, si certains éléments décisifs des dialogues shakespeariens ne sont effectivement pas rendus par les dialogues du film, ils s'y trouvent cependant incorporés par les images. Il s'agit, entre autres, des moments "explicatifs ", ceux-là même qui transformeraient le film en théâtre filmé s'ils étaient conservés tels

23 Akira Kurosawa, «A Personal Record : Kurosawa and I - Donald Richie, 1960 », in Akira Kurosawa - Interviews, Bert Cardullo (ed.), Jackson, U.P. of Mississippi, 2008, p. 15. Les propos entre crochets sont un ajout explicatif de Donald Richie.

24 Cette formule oxymorique est prononcée par la seconde sorcière à la scène i, lorsqu'elle répond à la première sorcière qui veut savoir quand elles se reverront : "SECOND WITCH. When the hurly-burly's done, / When the battle's lost and won. » (Macbeth, I.i.3-4). 
quels. Ainsi, en va-t-il des deux célèbres phrases de l'acte I : « Fair is foul, and foul is fair » (I.i.38), phrase énoncée par l'une des sorcières, et de la répartie de Macbeth à Banquo, juste avant que ces dernières ne leur apparaissent : " So foul and fair a day I have not seen. » (I.iii.36) Si dans le film Washizu prononce une phrase similaire, le rapport de contraste souligné par le propos entre la noirceur et la clarté qui révèle le caractère " ésotérique » et Unheimlich du moment, passe à l'image par trois formes de contraste de luminosités. D'abord celui entre l'aspect «nuit d'orage » en plein jour (les éclairs lancent leurs traces blafardes dans la noirceur du ciel), ensuite par le contraste entre l'ombre de la forêt et la blancheur du brouillard qui recouvre tout et, enfin, par le contraste des deux guerriers habillés de noir et cernés pas la forêt ténébreuse et la blancheur éclatante, quasi insoutenable, de l'apparition fantomatique rendue telle grâce à un éclairage violent de sa hutte qui donne une impression de surexposition de la pellicule.

En ce qui concerne cette apparition, plusieurs éléments sont à souligner. Tout d'abord, il s'agit d'une figure emblématique du répertoire Nô, tirée de la pièce Adachi ga hara (La Lande d'Adachi) ${ }^{25}$. Son apparition est reprise telle quelle dans le film, puisque ce fantôme féminin apparait en effet dans une hutte de bambous, en train de tisser, ou plus exactement en train de faire tourner un rouet pour on ne sait quel ouvrage en cours. Or, cette figure n'est pas reprise par hasard pour remplacer les «Weird Sisters", dénommées ainsi parce qu'elles rappellent notamment les Parques grecques, détentrices du Destin de l'humanité. Si dans la pièce c'est leur nombre qui « réveille » cette mémoire collective, dans le cas japonais, c'est l'image du rouet qui remplit cet office, en indiquant visuellement le fil de la vie qui se dévide avant qu'un coup de ciseau ne vienne finalement en interrompre le mouvement. De même, en ce qui concerne ce personnage, que l'on se souvienne de l'indécision qui plane sur le sexe des « Weird Sisters ». Macbeth les interpellant marque son étonnement en ces termes : « you should be women, / And yet your beard forbid me to interpret / That you are so. » (I.i.45-47). Cette hésitation est mise en perspective par le fait qu'à l'époque de Shakespeare les rôles féminins étaient joués par des jeunes hommes. Or, comme il a déjà été souligné, la tradition

25 Voir la traduction de cette pièce et sa notice, in Nô et Kyôgen II - Automne/Hiver, trad. René Sieffert, Paris, P.O.F., coll. Les Euvres capitales de la littérature japonaise, 1979, p. 114-128. 
théâtrale japonaise, aujourd'hui encore, reste exclusivement masculine. Ainsi, sur scène, le fantôme d'Adachi ga Hara est toujours interprété par un homme. Le personnage du film, quant à lui, remplit sa « fonction » d'indécision, à travers le grimage de ses traits, fortement « virilisés » par le maquillage, ainsi qu'à travers une modification de la voix qui ne permet plus de dire s'il s'agit d'une voix de femme ou d'homme. Cependant, là réside l'une des «trahisons » de Kurosawa vis-à-vis du Nô, car, malgré ces éléments d'indécision, c'est bien une femme (Naniwa Chieko) qui joue le rôle, virilisé, du fantôme.

Cet élément de «trahison » est d'importance, puisque la femme de Washizu, Asaji, aussi s'apparente à un «caractère » du Nô (la femme "d'âge moyen»), tout en détournant une fois encore la tradition. Si son visage est marqué, pour la plus grande partie du film, d'une impassibilité presque effrayante, qui la rapproche d'ailleurs (volontairement) du personnage de la sorcière, ses gestes, bien qu'inspirés du Nô, s'en détachent tout de même fortement. Et surtout, alors que Kurosawa a toujours clamé s'être exclusivement inspiré du Nô pour ce film, il s'avère que seuls les personnages féminins et les apparitions en sont le reflet. Les personnages masculins, eux, s'inspirent davantage du Kabuki, qu'il s'agisse des expressions de leurs visage ou de leurs mouvements, beaucoup trop amples et rapides pour provenir du Nô.

Pour en revenir à l'inquiétante rencontre avec la sorcière, le dialogue de la pièce, bien que raccourci et adapté aux besoins d'un film japonais $\mathrm{du} \mathrm{XX}^{\mathrm{e}}$ siècle, est fidèlement suivi par le réalisateur, notamment dans les propos échangés, par la suite, entre Macbeth et Banquo, qui ne savent pas trop s'ils doivent croire la prophétie ou non, s'en réjouir ou non. De même, c'est à ce moment du film que les premiers doutes de Washizu commencent à prendre corps, alors qu'il tente encore de contrôler les désirs secrets que recèle son cœur - et mis au jour de façon plus ou moins cryptique par l'apparition.

Pour ce qui concerne le conflit intérieur de Macbeth, c'est la tirade de la scène iii de l'acte I qui nous en donne l'image la plus vive :

If good, why do I yield to that suggestion, Whose horrid image doth unfix my hair, And make my seated heart knock at my ribs, Against the use of nature ? Present fears Are less than horrible imaginings.

My thought, whose murder yet is but fantastical, 
Shakes so my single state of man that function

Is smother'd in surmise, and nothing is

But what is not.

(I.iii.136-143)

La transposition, dans le film, est une fois de plus visuelle et vient suppléer au propos de la sorcière sur le «cœur étrange des hommes ». Il s'agit de l'animal totem imprimé sur la bannière de guerre de Washizu. Si, pour sa part, Miki, par ironie du sort (très aidé par le réalisateur), a pour animal totem le lièvre (animal certes agile et rapide, mais surtout doux et fragile), Washizu, lui, a pour emblème la scolopendre. Apparenté au mille-pattes, cet animal est si agile qu'il est difficile de l'attraper, mais, qui plus est, c'est un animal venimeux, qui paralyse immédiatement tout être vivant qui le touche, et le dévore voracement. Dès lors, si à ce moment du film Washizu semble encore vouloir suivre sa raison, avant que sa femme ne vienne définitivement balayer ses dernières réticences, sa bannière de guerre hausse les couleurs du fond véritable de son cœur - on pourrait dire son inconscient. On ne choisit pas un animal totem par hasard.

Avant de conclure, qu'il me soit permis de mettre encore en parallèle trois moments clés de la pièce, parmi d'autres, et leur transposition dans le film. Dans tous les cas, il s'agit de dialogues, ou monologues, fondamentaux pour la tragédie. Tout d'abord, on retrouve dans le film deux mises en image particulièrement fortes de la tirade de Lady Macbeth, lorsqu'elle décide de pousser son mari au meurtre de Duncan. Le vocabulaire de la tirade est précis :

The raven himself is hoarse

That croaks the fatal entrance of Duncan

Under my battlements. Come, you spirits

That tend on mortal thoughts, unsex me here,

And fill me from the crown to the toe, top-full

Of direst cruelty. Make thick my blood,

Stop up th'access and passage to remorse,

That no compunctious visitings of nature

Shake my fell purpose, nor keep peace between

Th'effect and it. Come to my woman's breasts,

And take my milk for gall, you murth'ring ministers,

Wherever, in your sightless substances,

You wait on nature's mischief. Come, thick Night,

And pall thee in the dunnest smoke of Hell,

That my keen knife see not the wound it makes,

Nor Heaven peep through the blanket of the dark,

To cry "hold, hold!" 
Ce monologue se trouve «traduit» par deux effets visuels formant parenthèses autour du meurtre du Seigneur Fujimaki. Le premier, se situe avant le meurtre, à travers la scène liée au stratagème du vin (sake) trafiqué aux somnifères pour endormir les gardes. À ce moment, Asaji pénètre dans une salle dont l'intérieur est si opaque qu'elle y est littéralement happée un instant, avant qu'elle n'en réémerge avec le vin en question. Pendant un court instant le spectateur a le sentiment que le personnage entre de plain-pied dans le néant lui-même, dont le personnage serait parfaitement familier. Le second, se situe après le meurtre, alors que le couple est réuni dans la chambre interdite (celle où le traître dont Washizu a hérité s'est suicidé par seppuku). Le réalisateur intercale une courte séquence montrant un ciel d'une noirceur d'encre sur lequel se détache avec peine un fin croissant de lune blafard et devant lequel passe fugitivement un corbeau poussant un cri lugubre. Ce croissant de lune a plusieurs significations. Tout d'abord il rappelle le blason du Seigneur Fujimaki qui vient d'être assassiné; ensuite il fait évidemment référence au corbeau de la tirade de Lady Macbeth; enfin, on peut y lire aussi un clin d'œil à la première scène du Chien andalou de Luis Buñuel, qui opère un parallèle entre la lune coupée par un nuage effilé et un œil de femme tranché au rasoir. Chez Kurosawa, c'est le noir corbeau qui traverse la lune et qui, par le lien subliminal noué avec la scène de Buñuel, métaphorise pour le spectateur ce qui vient d'avoir lieu hors champ (comme c'est d'ailleurs le cas dans la pièce, où le meurtre a lieu en coulisses). Précisons, pour ce qui est des corbeaux, très présents dans le film, qu'à l'instar de la signification démoniaque que la culture populaire occidentale leur prêtait à l'époque de la pièce, de même, au Japon, les corbeaux étaient considérés comme les suppôts des démons, et étaient susceptibles de servir d'intermédiaires entre les vivants et les morts.

La transposition suivante concerne les signes « contre nature » qui ont annoncé, sans que personne ne les interprète correctement, le meurtre de Duncan. Dans le film, ces signes sont déplacés sur le meurtre de Miki/Banquo. Cependant, c'est bien le texte shakespearien qui fournit la trame de la mise en scène. C'est le dialogue entre Ross et un « vieil homme » pour qui, « Threescore and ten [...] this sore night / Hath trifled former knowings » (II.iv.1-4), qui en fournit les éléments. Ross répond en effet : 




Dans le film, Kurosawa utilise cette indication dans la scène où Miki et son fils sont censés se préparer pour assister, en tant qu'hôtes d'honneurs, à la réception que donne Washizu le soir même. Un seul cheval suffit dans la mise en scène du réalisateur, celui de Miki qui, parce que soudainement " ensauvagé », refuse de façon inattendue de se laisser seller. Quand bien même son fils y lit un mauvais augure, Miki refuse de l'écouter et force ses serviteurs à seller son étalon. Acte par lequel, du même geste, son propre Destin se trouve scellé. Dans les deux cas, les signes, tout similaires, ne sont pas vus/écoutés.

Enfin, avant de conclure, et pour faire une brève incursion du côté de Ran, qui suit les mêmes principes de déplacements que Kumonosu Jô dans la transposition de la pièce à l'écran, un seul exemple sera ici sollicité afin de mettre en lumière cette mise en pratique systématique du cinéaste. Kurosawa ayant dû opter pour trois enfants mâles en lieu et place des trois filles du roi Lear pour des raisons toutes pratiques de crédibilité des événements (à l'époque où se situe la pièce et l'action du film il aurait été impensable que des filles héritent), il emploie cependant une figure féminine (Dame Kaede) qui condense en elle les traits les plus forts, et surtout les plus cruels, de Regan certes, mais surtout de Goneril (c'est évident lorsque l'on confronte le texte shakespearien au film). Or, là encore, ce n'est pas seulement à travers la psychologie du personnage que ces traits sont révélés au spectateur. Réutilisant le même stratagème de mise en scène employé pour Asaji dans Kumonosu Jô, le personnage de Kaede est dépeint sous les traits d'un personnage de Nô. Même "masquemaquillage », même genre de kimono. Mais, comme toujours, c'est le texte qui impose sa volonté au film. En effet, deux éléments se conjoignent, qui octroient toute sa force au personnage. Dans la pièce de Shakespeare, Goneril est par deux fois comparée à une renarde, par exemple, dès l'acte I, scène iv, dans une chanson du fou de Lear :

A fox, when one has caught her,

And such a daughter,

Should sure to the slaughter, 
If my cap would buy a halter, So the Fool follows after.

(King Lear, I.iv.72)

Or, au Japon, comme en Europe, le renard est mythiquement connu pour être dangereux, et surtout pour savoir aveugler les hommes afin de les mener à la déchéance (souvent, d'ailleurs, sous la forme de renardes). Par ailleurs, pour rester dans le registre animal, Goneril est aussi plusieurs fois comparée à un serpent par son père. Par exemple, comme dans la scène ii de l'acte II, au moment où Regan tente de se débarrasser de son père en le renvoyant auprès de Goneril, la réponse de Lear est sans appel :

Never, Regan.

She hath abated me of half my train,

Look'd black upon me, struck me with her tongue,

Most serpent-like, upon the very heart.

All the stor'd vengeances of heaven fall

On her ingrateful top ! Strike her Young bones,

You taking airs, with lameness !

(King Lear, II.ii.122)

C'est de ce «serpent-like» que Kurosawa tire parti, depuis le théâtre Nô, afin de donner tout son éclat à la froideur du personnage de Dame Kaede. Ainsi, c'est en grande partie sur le kimono du personnage que repose l'aspect reptilien de ce dernier. S'inspirant du personnage de démon-serpent de la célèbre pièce Dôjôji (en anglais snake-lady, comme on dit Lady Kaede dans la traduction anglaise du nom du personnage du film), Kurosawa attribue à la figure de Kaede un kimono qui répond exactement à la description que donne Donald Keene de celui du personnage dans la pièce japonaise : " the snake-lady in Dôjôji always wears a robe with a lozenge pattern suggesting a snakeskin ${ }^{26}$ ». Par suite, la relation entre cette figure de serpent et celle de la femmerenarde évoquée plus haut, aussi séductrice et mortelle que la femmeserpent, est établie dans une scène où l'homme de main de Jirô doit prouver qu'il a bien exécuté, sur les instances de Kaede, la femme de son maître, jusqu'alors simplement répudiée par ce dernier. L'homme de main devant normalement rapporter la tête de la première femme, substitue à celle-ci une tête de renard en pierre. Cherchant à ouvrir les

\footnotetext{
${ }^{26}$ Donald Keene, "Nô the Classical Theatre of Japan ", in Nô and Bunraku, p. 66. Dans le film de Kurosawa, le design du vêtement est non seulement en losange mais aussi gris argenté, ce qui renforce l'aspect reptilien de Dame Kaede lorsqu'elle se déplace. Précisons par ailleurs que Dame Kaede devient la concubine du deuxième fils de Hidetora/Lear (Jirô) lorsque son mari (frère aîné de Jirô) est assassiné par l'homme de main de son frère cadet.
} 
yeux de son maître sur les dangers qu'il court à trop écouter Dame Kaede, il en profite alors pour lui adresser un discours dans lequel il lui rappelle que les renards prennent souvent l'aspect de femmes belles et enivrantes, qui finissent par mener les hommes qui les écoutent à leur perte. Bien évidemment, à ce moment précis, et en raison des actions antérieures de Kaede, le message est limpide, aussi bien pour les personnages qui l'écoutent que pour les spectateurs. Cependant, Jirô ne saura pas écouter son fidèle serviteur, se laissera piéger par sa maîtresse et y trouvera la mort. Toute la mise en scène de Kurosawa rejoue ainsi, en les déplaçant à des fins de condensation de l'action, les relations et les jalousies qui se jouent entre Goneril et Regan d'une part, Albany, Cornouailles et le bâtard Edmond d'autre part.

En conclusion, on le voit, les critiques formulées à l'encontre de Kurosawa, selon lesquelles ses adaptions de Shakespeare ne seraient pas « fidèles » à la lettre shakespearienne, s'effondrent d'elles-mêmes dès lors que l'on s'attache, de façon attentive, aux éléments constitutifs $\mathrm{du}$ tragique du dramaturge élisabéthain. La première raison vient de son refus de faire du théâtre filmé. La seconde ressortit davantage de la culture asiatique en général : nous sommes en ces territoires hors des cultures du Logos. La parole n'a absolument pas, dans ces civilisations, la valeur que peuvent lui accorder les occidentaux. Dès lors, l'art cinématographique qui en émerge n'a pas pour ambition d'y puiser ses ressources. Kurosawa a ainsi cherché à traduire visuellement ce que suggérait le texte shakespearien et qui, repris tel quel, serait devenu redondant. Le parti qu'il a tiré du Nô et du Kabuki, a justement été de déplacer le Logos du côté du mouvement scénique afin de supprimer tout ce qui devenait superflu à la caméra. Procédant ainsi, il est parvenu à ranimer et redynamiser la pratique de la mise en scène qui avait cours à l'époque du dramaturge. En prenant certaines distances vis-à-vis du verbe des tragédies élisabéthaines, il est finalement resté fidèle à celles-ci par jeux de déplacements, coupures et remontages. Ainsi, les stratégies de mise en scène suivies par le réalisateur, sur lesquelles se sont entées les traditions théâtrales japonaises, ont pour effet de concentrer à travers elles l'essence de la tragédie. Nô et Kabuki ne se sont donc pas substitués à la dramaturgie shakespearienne, mais 
au contraire ont permis d'en exhausser l'essence tragique qui pouvait, à travers eux, être transposée à l'écran.

Olivier AMMOUR-MAYEUR

Université Rikkyo - Tokyo 